Ágora Rev. Cient. 2019; 06(01):e6

\title{
Valoración del estado nutricional de recién nacidos por el método de Metcoff
}

\author{
Assessing nutritional status of newborns by Metcoff's method \\ Marita Isenia García Alberca ${ }^{1}$, Clever Humberto Leiva Herrada ${ }^{1}$, Elias José Leiva Parra ${ }^{1}$
}

\section{RESUMEN}

Introducción: Si bien los parámetros antropométricos (peso, talla y perímetro cefálico) usados en la evaluación nutricional del recién nacido (RN) pueden ser normales, algunos RN a término (RNT) adecuados para su edad gestacional (AEG) sufren de desnutrición fetal cuando se valoran los compartimientos graso y proteico a través del Método de Metcoff. Objetivo: Se buscó determinar el estado nutricional de los RNT Adecuados para la Edad Gestacional mediante la evaluación clínica empleando el método de Metcoff en el servicio de Neonatología del Hospital de Sullana. Diciembre 2011 - Enero 2012. Materiales y Métodos: Se realizó un estudio prospectivo, transversal, descriptivo y observacional en el Hospital de Sullana. Se evaluó el estado nutricional de una muestra de 93 RNT AEG, vivos, de parto eutócico y único, sin la presencia de factores de riesgo que afecten la salud de la madre, el RN o ambos. La selección de la muestra fue probabilísticade tipo accidental, requiriendo para un nivel de confianza del 95\%, 96 RN.Los resultados se muestran en tablas y gráficos. Resultados: Se evaluaron 93 RNT AEG. Predominó el sexo masculino (50.5 $\%$ ) con respecto al femenino (49.5\%), al igual que en los desnutridos $(66.7 \%)$ frente al $47.4 \%$ de los nutridos. El promedio y desviación estándar del peso, talla, perímetro cefálico y perímetro braquial para los considerados como nutridos fue $3282.2 \pm 325.3 \mathrm{~g}, 49.12 \pm 1.54 \mathrm{~cm}, 33.67 \pm 1.23$ y $10.36 \pm 0.67 \mathrm{~cm}$, respectivamente, mientras que para los desnutridos fue $2972.7 \pm 268.2 \mathrm{~g}, 48.7 \pm 2.01 \mathrm{~cm}, 33.03 \pm 1.34 \mathrm{~cm}$ y $9.71 \pm 0.68$, respectivamente. Conclusión: El método de Metcoff mostró desnutrición clínica en 15 RNT AEG, lo que representa una incidencia del $16.1 \%$.

Palabras claves: recién nacido a término, edad gestacional, estado nutricional, Método de Metcoff, desnutrición fetal.

\begin{abstract}
Introduction: Despite anthropometric parameters (weight, length and head circumference) used in the nutritional evaluation of newborn can be normal; nevertheless, some newborns delivered at term catalogued like appropiate for gestational age (AGA), suffer from fetal malnutrition (FM) when the compartments are valued greasy and protein through Metcoff's test. Objective: the incidence of FM in term newborns AGA, using clinical assessment of nutritional status at birth and its qualification according to Metcoff, from December 2011 - January 2012 in Hospital of Sullana. Materials and Methods: A prospective, crosssectional, descriptive, and mm study was made in the Hospital de Sullana. The nutritional state was evaluated of a sample of 93 newborns delivered at term AGA, alive, eutocic and singleton delivery, without the presence of risk factors that affect the health of the mother, the newborn or both. The selection was of probabilistic sample of type accidental. Results are shown in tables and graphs. Results: 93 newborns delivered at term AGA were evaluated. There was predominance of male sex $(50.5 \%)$ with respect to the female sex $(49.5 \%)$. The mean values and standard deviations of the weight, length, head circumference and circumference for the considered ones as nourished well were as follows: $3282.2 \pm 325.3 \mathrm{~g}, 49.12 \pm 1.54 \mathrm{~cm}$, $33.67 \pm 1.23$ y $10.36 \pm 0.67 \mathrm{~cm}$, respectively, whereas for the undernourished ones were $2972.7 \pm 268.2 \mathrm{~g}$, $48.7 \pm 2.01 \mathrm{~cm}, 33.03 \pm 1.34 \mathrm{~cm}$ y $9.71 \pm 0.68$. Conclusion: Metcoff's test showed clinical fetal malnutrition in 15 term newborns AGA, which represents an incidence of $16 \%$.
\end{abstract}

Keywords: newborn term, gestational age, nutritional status, Metcoff's test, fetal malnutrition.

${ }^{1}$ Servicio de Neonatología. Departamento de Pediatría. Hospital de Sullana II-2. Ministerio de Salud. Piura - Perú. 


\section{INTRODUCCIÓN}

El término desnutrición fetal puede ser encontrado en cualquier país, cultura y nivel socioeconómico. Este es un diagnóstico clínico totalmente independiente del peso al nacer del neonato. La desnutrición en diferentes momentos de la vida temprana produce diferentes efectos y deja marcas permanentes en la fisiología celular y molecular y en la morfología de ciertos órganos y tejidos (1-7).

Si bien los parámetros antropométricos (peso, talla y perímetro cefálico) usados en la evaluación nutricional del recién nacido $(\mathrm{RN})$ pueden ser normales, algunos RN a término (RNT) adecuados para su edad gestacional (AEG) sufren de desnutrición fetal cuando se valoran los compartimientos graso y proteico a través del Método de Metcoff. El método de Metcoff evalúa 9 signos del estado nutricional, calificándose cada uno desde 4 hasta 1 . Siendo el 4 indicador de que no hay desnutrición y el 1 de que sí hay signos claros de desnutrición fetal. Al sumar los puntajes existe un puntaje de 9 - 36. Un puntaje menor o igual a 24 indica un diagnóstico positivo de desnutrición fetal $(8,12,13)$.

Uno de los problemas de salud más importantes en el Perú es la desnutrición, siendo los grupos más vulnerables los niños y las mujeres gestantes $(10,18,19)$. En un estudio que se realizó en nueve hospitales del Ministerio de Salud de las tres regiones naturales, se encontró que los factores de riesgo que tuvieron alto valor predictivo como causa de mortalidad perinatal fueron los relacionados directamente con el recién nacido: bajo peso, prematuridad, depresión al nacer, morbilidad neonatal y desnutrición intrauterina (15). La importancia de conocer el estado de salud y bienestar en que se encuentran los neonatos desde el punto de vista nutricional radica, en que, el solo hecho de ser desnutridos los hace recién nacidos de alto riesgo lo que puede incrementar la morbimortalidad en la vida postnatal.

La desnutrición fetal que ocurre en un embarazo aparentemente no complicado, en una madre aparentemente sana y con bebes desnutridos clínicamente pide una explicación, así como una prevención. Estos neonatos pueden o no ser identificados y pasar desapercibidos sin ser diagnosticados a tiempo, siendo muy probable que estos vuelvan al hospital por problemas de desnutrición, ya sea a corto, mediano y largo plazo. La importancia de conocer el estado de salud y bienestar en que se encuentran los neonatos desde el punto de vista nutricional radica, en que, el solo hecho de ser desnutridos los hace recién nacidos de alto riesgo lo que puede incrementar la morbimortalidad en la vida postnatal $(14,16)$. Por esta razón, se tuvo como objetivo determinar el estado nutricional de los RNAT Adecuados para la Edad Gestacional mediante la evaluación clínica empleando el método de Metcoff.

\section{MATERIALES Y MÉTODOS}

El presente estudio tuvo como escenario de investigación el Servicio de Neonatología, departamento de Pediatría del Hospital de Sullana, II-2 MINSA - Perú, durante los meses Diciembre 2011 - Enero 2012. Se realizó un estudio prospectivo, transversal, descriptivo y observacional. Los criterios de inclusión fueron: 1) RNAT de 37 a 41 semanas Adecuados para la Edad Gestacional cuyo nacimiento fue por vía vaginal, único y que haya ocurrido en el hospital de Sullana II - 2 MINSA: 2) RNAT de 37 a 41 semanas Adecuados para la Edad Gestacional, sin patología, sin complicaciones durante el parto (depresión, asfixia, líquido amniótico meconial, circular de cordón, RPM mayor de 24 horas); 3 ) RNAT de 37 a 41 semanas Adecuados para la Edad Gestacional no hospitalizados; 4) RNAT de 37 a 41 semanas Adecuados para la Edad Gestacional sin malformaciones congénitas mayores. Los criterios de exclusión fueron: 1) Recién nacidos producto de embarazo Gemelar; 2) Recién nacidos con riesgo: Sepsis neonatal, depresión al nacer; 3) Hijos de madres con complicaciones o intercurrencias durante el embarazo: HTT, preeclampsia, ITU); 4) Recién nacidos de madres que no tenían exámenes auxiliares prenatales.

La población estuvo constituida por todos los recién nacidos a término adecuados para la edad gestacional productos de parto eutócico durante el periodo de estudio. El tamaño de la muestra para el estudio fue de 93; con $Z=$ Nivel de fiabilidad de $95 \%$ (valor estándar de 1,96) y e = Margen de error de las estimaciones. En el estudio, se asume un error del 5\% (valor estándar de 0,05$)$. Luego de la recolección de datos, estos fueron codificados y depurados, a través de la base de datos del programa SPSS 15.0.En este programa se procesó toda la información y se realizó las pruebas estadísticas correspondientes: intervalos confidenciales para las medias poblacionales, la prueba t-student para la diferencia de medias y la prueba exacta de Fisher para determinar la relación de las variables. 


\section{RESULTADOS}

La Tabla 1 muestra el total de recién nacidos a término, clasificados según su peso al nacer, de los cuales el 3.7\% (14) fueron PEG, 94. \% (357) AEG (94.4\%) y $1.9 \%$ (7) GEG.

En cuanto al sexo (Tabla 2) el 49.5\% (46) correspondían al sexo femenino y el 50.5\% (47) al sexo masculino. Según grupo de recién nacidos en los desnutridos el $66.7 \%$ fue de sexo masculino y el $33.3 \%$ de sexo femenino. En los nutridos el $52.6 \%$ de sexo femenino y el $47.4 \%$ de sexo masculino. Según la Prueba exacta de Fisher no hay relación significativa entre las categorías de las variables $(\mathrm{P}=0.260)$. Del total $16 \%$ (15) presenta signos de desnutrición clínica y el $84 \%$ (78) no presentan.

En la Tabla 3 se observa que del total de desnutridos según Metcoff el $33.3 \%$ (5) corresponden a hijos de madres adolescentes, en comparación al $10.3 \%$ (8) de los nutridos. El $53.3 \%$ de los recién nacidos desnutridos corresponden a madres adultas, mientras que para los nutridos es el $75.6 \%$ (59); en cuanto a las madres añosas corresponde el $13.3 \%$ (2) y 14.1 (11) para las desnutridos y nutridos respectivamente. Según la prueba exacta de Fisher, existe una relación marginalmente significativa entre las categorías de las variables $(\mathrm{P}=0.057)$.

La Tabla 4 muestra el estado nutricional según Metcoff y talla materna. En cuanto a los recién nacidos desnutridos el $53.3 \%$ corresponden a madres con talla $<1.50 \mathrm{~cm}$, el $33.3 \%$ a madres con talla entre $1.50-1.55$, el $20 \%$ entre $1.56-1.60$ y en madres cuya talla es mayor de $1.60(0 \%)$. Para los nutridos el $35.9 \%, 32.1 \%, 25.6 \%$ y $6.4 \%$ corresponden a madres cuyas tallas están entre $<1.50, \quad 1.50-1.55, \quad 1.56-1.60$ y $>1.60 \quad \mathrm{~cm}$ respectivamente. Según la Prueba exacta de Fisher no hay relación significativa entre las categorías de las variables $(\mathrm{P}=0.848)$.

En cuanto al IMC pregestacional (Tabla 5), se observa que el $6.4 \%$ (2) de los desnutridos según Metcoff corresponden a hijos de madres con bajo peso, el $69.2 \%$ (10) a madres con peso ideal, el $15.4 \%$ a madres con sobrepeso y el $9 \%$ (1) a madres con obesidad. Con respecto a los nutridos el $13.3 \%$ corresponden a madres con bajo peso, el $66.7 \%$ a madres con peso ideal, el $13.3 \%$ a madres con sobrepeso y el $6.7 \%$ de los recién nacidos nutridos a madres con obesidad. Según la Prueba exacta de Fisher no hay relación significativa entre las categorías de las variables $(\mathrm{P}=0.814)$.

Con respecto a la ganancia ponderal materna (GP) (Tabla 6), se observa que del total de desnutridos según Metcoff, el 33.3\% son hijos de madres cuya GP durante la gestación fue $<9 \mathrm{~kg}$, el $26.7 \%$ hijos de madres con una GP entre 9 y $12 \mathrm{~kg}$ y un mayor porcentaje $40 \%$ corresponden a hijos de madres cuya GP fue $>12 \mathrm{~kg}$. Para los nutridos, el $43.6 \%, 26.9 \%$ y el $29.5 \%$ corresponden a recién nacidos de madres cuya GP fue: $\langle 9 \mathrm{~kg}, 9-12 \mathrm{~kg}\rangle$ $12 \mathrm{~kg}$ respectivamente. Según la Prueba exacta de Fisher no hay relación significativa entre las categorías de las variables $(\mathrm{P}=0.679)$.

En cuanto al grado de instrucción de la madre (Tabla 7), podemos apreciar que del total de desnutridos según Metcoff el 6.7\% (1) de los recién nacidos corresponden a madres analfabetas al igual que el nivel superior, el $33.3 \%$ (5) a madres con educación primaria y el $53.3 \%$ (8) hijos de madres con educación secundaria. Con respecto a los nutridos el $2.6 \%$ (2), 19.2\% (15), $64.1 \%$ (50) y el $14.1 \%$ (11) corresponden a recién nacidos de madres analfabetas, con educación primaria, secundaria y superior respectivamente. Según la Prueba exacta de Fisher no hay relación significativa entre las categorías de las variables $(\mathrm{P}=0.397)$.

En la Tabla 8 observamos el control prenatal materno en relación al estado nutricional según Metcoff. Del total de desnutridos, el 13.3\% (2) de los recién nacidos corresponden a madres no controladas (CPN menos de 4) y el $86.7 \%$ (13) corresponden a madres controladas (más de 4 CPN). En cuanto a los nutridos el $15.4 \%$ (12) corresponden a madres no controladas y el $84.6 \%$ (66) a madres controladas. Según la Prueba exacta de Fisher no hay relación significativa entre las categorías de las variables $(\mathrm{P}=0.841)$.

En la Tabla 9 se detalla el promedio y desviación estándar del peso, talla, perímetro cefálico y perímetro braquial para los considerados como nutridos es $3282.2 \pm 325.3 \mathrm{~g}$, $49.12 \pm 1.54 \mathrm{~cm}, 33.67 \pm 1.23$ y $10.36 \pm 0.67 \mathrm{~cm}$ respectivamente, mientras que para los desnutridos es $2972.7 \pm 268.2 \mathrm{~g}, 48.7 \pm 2.01 \mathrm{~cm}$, $33.03 \pm 1.34 \mathrm{~cm}$ y $9.71 \pm 0.68$, respectivamente.

Finalmente, en la Tabla 10 observamos el número de casos de acuerdo al puntaje obtenido mediante la evaluación clínica del estado nutricional según el método de Metcoff. Del total de recién nacidos desnutridos, el $1.1 \%$ (1) tuvo 19 puntos al igual que los que tuvieron 21 puntos (1), $3.2 \%$ (3) 22 puntos, $7.5 \%$ (7) 23 puntos y el $3.2 \%$ (3) 24 puntos. En cuanto al grupo de los nutridos el mayor porcentaje lo observamos a en los recién nacidos que obtuvieron 30 puntos en el 17.2 (16), seguida del $15.1 \%$ (14) con 27 puntos y el $13.9 \%$ (28) con 28 puntos. 
Tabla 1. Clasificación de los reción nacidos a término según su peso al nacer y edad gestacional. Servicio de Neonatología del Hospital de Sullana II-2 MINSA. Diciembre 2011 - Enero 2012.

\begin{tabular}{|c|c|c|}
\hline $\begin{array}{c}\text { RECIÉN NACIDOS } \\
\text { A TÉRMINO }\end{array}$ & N & PORCENTAJE \\
\hline PEG & 14 & $3.7 \%$ \\
\hline AEG & 357 & $94.4 \%$ \\
\hline GEG & 7 & $1.9 \%$ \\
\hline Total & 378 & $100.0 \%$ \\
\hline
\end{tabular}

FUENTE: Base de datos

Tabla 2. Sexo de los recién nacidos nutridos y desnutridos según Metcoff. Servicio de Neonatología del Hospital de Sullana II-2 MINSA. Diciembre 2011 - Enero 2012.

\begin{tabular}{|c|c|c|c|c|c|}
\hline \multicolumn{6}{|c|}{ Evaluación Clínica Nutricional: Método de Metcoff } \\
\hline \multirow[b]{2}{*}{ Sexo } & \multicolumn{2}{|c|}{ Desnutridos } & \multicolumn{2}{|c|}{ Nutridos } & \multirow[b]{2}{*}{ Total } \\
\hline & $\mathbf{n}$ & n \% & $\mathbf{N}$ & n \% & \\
\hline Femenino & 5 & $33.3 \%$ & 41 & $52.6 \%$ & $46(49.5 \%)$ \\
\hline Masculino & 10 & $66.7 \%$ & 37 & $47.4 \%$ & $47(50.5 \%)$ \\
\hline Total & 15 & $100.0 \%$ & 78 & $100.0 \%$ & $93(100.00 \%)$ \\
\hline
\end{tabular}

FUENTE: Base de datos

Prueba exacta de Fisher: $1.893 \quad \mathrm{P}=0.260$

Tabla 3. Edad materna y estado nutricional según Metcoff. Servicio de Neonatología del Hospital de Sullana II-2 MINSA. Diciembre 2011 - Enero 2012.

\begin{tabular}{|c|c|c|c|c|c|}
\hline \multirow[t]{2}{*}{ EDAD } & \multicolumn{2}{|c|}{ Desnutridos } & \multicolumn{2}{|c|}{ Nutridos } & \multirow[b]{2}{*}{ Total } \\
\hline & $n$ & $\%$ & $\mathrm{n}$ & $\%$ & \\
\hline $\begin{array}{l}\leq 19 \\
\text { (adolescentes) }\end{array}$ & 5 & $33.3 \%$ & 8 & $10.3 \%$ & $13(14 \%)^{\star}$ \\
\hline $20-34$ (adultas) & 8 & $53.3 \%$ & 59 & $75.6 \%$ & $67(72 \%)$ \\
\hline$>=35$ (añosas) & 2 & $13.3 \%$ & 11 & $14.1 \%$ & $13(14 \%)$ \\
\hline Total & 15 & $100.0 \%$ & 78 & $100.0 \%$ & $100(100 \%)$ \\
\hline \multicolumn{6}{|l|}{ uente: Base de datos } \\
\hline
\end{tabular}

Tabla 4. Talla materna y estado nutricional según Metcoff. Servicio de Neonatología del Hospital de Sullana II-2 MINSA. Diciembre 2011 - Enero 2012.

\begin{tabular}{|l|c|c|c|c|c|}
\hline \multirow{2}{*}{$\begin{array}{c}\text { TALLA } \\
\text { MATERNA }\end{array}$} & \multicolumn{2}{|c|}{ Desnutridos } & \multicolumn{2}{|c|}{ Nutridos } & \multirow{2}{*}{ Total } \\
\cline { 2 - 5 } & $\mathbf{N}$ & $\%$ & $\mathbf{N}$ & $\%$ & \\
\hline$<1.50$ & 8 & $53.3 \%$ & 28 & $35.9 \%$ & $36(38.7 \%)$ \\
\hline $1.50-1.55$ & 4 & $26.7 \%$ & 25 & $32.1 \%$ & $29(31.2 \%)$ \\
\hline $1.56-1.60$ & 3 & $20.0 \%$ & 20 & $25.6 \%$ & $23(24.7 \%)$ \\
\hline$>1.60$ & 0 & $0 \%$ & 5 & $6.4 \%$ & $5(5.4 \%)$ \\
\hline Total & 15 & $100.0 \%$ & 78 & $100.0 \%$ & $100(100 \%)$ \\
\hline
\end{tabular}

Fuente: Base de datos

Prueba exacta de Fisher: $1.539 \quad \mathrm{P}=0.848$ 
Tabla 5. IMC pregestacional y estado nutricional según Metcoff. Servicio de Neonatología del Hospital de Sullana II-2 MINSA. Diciembre 2011 - Enero 2012.

\begin{tabular}{l|c|c|c|c|c|}
\hline \multirow{2}{*}{$\begin{array}{c}\text { IMC } \\
\text { PREGESTACIONAL }\end{array}$} & \multicolumn{2}{|c|}{ Desnutridos } & \multicolumn{2}{c|}{ Nutridos } & \multirow{2}{*}{ Total } \\
\cline { 2 - 5 } & $\mathbf{N}$ & $\%$ & $\mathbf{N}$ & $\%$ & \\
\hline Bajo peso & 2 & $13.3 \%$ & 5 & $6.4 \%$ & $7(7.5 \%)$ \\
\hline Peso ideal & 10 & $66.7 \%$ & 54 & $69.2 \%$ & $64(68.8 \%)$ \\
\hline Sobrepeso & 2 & $13.3 \%$ & 12 & $15.4 \%$ & $14(15 \%)$ \\
\hline Obesidad & 1 & $6.7 \%$ & 7 & $9.0 \%$ & $8(8.6 \%)$ \\
\hline Total & 15 & $100.0 \%$ & 78 & $100.0 \%$ & $100(100 \%)$ \\
\hline
\end{tabular}
Fuente: Base de datos
Prueba exacta de Fisher: $1.234 \quad \mathrm{P}=0.814$

Tabla 6. Ganancia ponderal materna y estado nutricional según Metcoff. Servicio de Neonatología del Hospital de Sullana II-2 MINSA. Diciembre 2011 - Enero 2012.

\begin{tabular}{|c|c|c|c|c|c|}
\hline \multirow{2}{*}{$\begin{array}{c}\text { GANANCIA } \\
\text { PONDERAL }\end{array}$} & \multicolumn{2}{|c|}{ Desnutridos } & \multicolumn{2}{|c|}{ Nutridos } & \multirow{2}{*}{ Total } \\
\cline { 2 - 5 } & $\mathbf{N}$ & $\%$ & $\mathbf{N}$ & $\%$ & \\
\hline$<9 \mathrm{~kg}$ & 5 & $33.3 \%$ & 34 & $43.6 \%$ & $39(41.9 \%)$ \\
\hline $9-12 \mathrm{~kg}$ & 4 & $26.7 \%$ & 21 & $26.9 \%$ & $25(26.9 \%)$ \\
\hline$>12 \mathrm{~kg}$ & 6 & $40.0 \%$ & 23 & $29.5 \%$ & $29(31.2 \%)$ \\
\hline Total & 15 & $100.0 \%$ & 78 & $100.0 \%$ & $93(100 \%)$ \\
\hline
\end{tabular}

Fuente: Base de datos

Prueba exacta de Fisher: $0.842 \quad \mathrm{P}=0.679$

Tabla 7. Grado de instrucción y estado nutricional según Metcoff. Servicio de Neonatología del Hospital de Sullana II-2 MINSA. Diciembre 2011 - Enero 2012.

\begin{tabular}{|l|c|c|c|c|c|}
\hline \multirow{2}{*}{$\begin{array}{c}\text { GRADO DE } \\
\text { INSTRUCCIÓN }\end{array}$} & \multicolumn{2}{|c|}{ Desnutridos } & \multicolumn{2}{|c|}{ Nutridos } & \multirow{2}{*}{ Total } \\
\cline { 2 - 5 } & $\mathbf{N}$ & $\%$ & $\mathbf{N}$ & $\%$ & \\
\hline Analfabeta & 1 & $6.7 \%$ & 2 & $2.6 \%$ & $3(3.2 \%)$ \\
\hline Primaria & 5 & $33.3 \%$ & 15 & $19.2 \%$ & $20(21.5 \%)$ \\
\hline Secundaria & 8 & $53.3 \%$ & 50 & $64.1 \%$ & $58(62.4 \%)$ \\
\hline Superior & 1 & $6.7 \%$ & 11 & $14.1 \%$ & $12(13 \%)$ \\
\hline Total & 15 & $100.0 \%$ & 78 & $100.0 \%$ & $100(100 \%)$ \\
\hline
\end{tabular}

Fuente: Base de datos

Prueba exacta de Fisher: $2.962 \quad \mathrm{P}=0.397$

Tabla 8. Control prenatal materno y estado nutricional según Metcoff. Servicio de Neonatología del Hospital de Sullana II-2 MINSA. Diciembre 2011 - Enero 2012.

\begin{tabular}{|l|c|c|c|c|c|}
\hline \multirow{2}{*}{$\begin{array}{l}\text { CONTROLE } \\
\text { PRENATAL }\end{array}$} & \multicolumn{2}{|c|}{ Desnutridos } & \multicolumn{2}{c|}{ Nutridos } & \multirow{2}{*}{ Total } \\
\cline { 2 - 5 } & $\mathbf{N}$ & $\%$ & $\mathbf{N}$ & $\%$ & \\
\hline No controlada & 2 & $13.3 \%$ & 12 & $15.4 \%$ & $14(15 \%)$ \\
\hline Controlada & 13 & $86.7 \%$ & 66 & $84.6 \%$ & $79(84.9 \%)$ \\
\hline Total & 15 & $100.0 \%$ & 78 & $100.0 \% \%$ & $100(100 \%)$ \\
\hline
\end{tabular}

Fuente: Base de datos

Prueba exacta de Fisher: $0.043 \quad P=0.837$ 
Tabla 9. Parámetros antropométricos de los recién nacidos nutridos y desnutridos según Metcoff. Servicio de Neonatología del Hospital de Sullana II-2 MINSA. Diciembre 2011 - Enero 2012.

\begin{tabular}{|l|c|c|c|}
\hline \multirow{2}{*}{ Variables } & \multicolumn{2}{|c|}{$\begin{array}{c}\text { Evaluación nutricional } \\
\text { (Promedio +l- D.E) }\end{array}$} & P \\
\cline { 2 - 4 } & $\begin{array}{c}\text { Desnutridos } \\
\mathbf{n}=\mathbf{1 5}\end{array}$ & $\begin{array}{c}\text { Nutridos } \\
\mathbf{n = 7 8}\end{array}$ & Valor * \\
\hline Peso (gr) & $2972.7 \pm 268.2$ & $3282.2 \pm 325.3$ & $0.001^{\star \star}$ \\
\hline Talla (cm) & $48.7 \pm 2.01$ & $49.12 \pm 1.54$ & 0.406 \\
\hline $\begin{array}{l}\text { Perímetro } \\
\text { cefálico (cm) }\end{array}$ & $33.03 \pm 1.34$ & $33.67 \pm 1.23$ & 0.075 \\
\hline $\begin{array}{l}\text { Perímetro } \\
\text { braquial }(\mathbf{c m})\end{array}$ & $9.71 \pm 0.68$ & $10.36 \pm 0.67$ & $0.001^{\star}$ \\
\hline
\end{tabular}

FUENTE: Base de datos

* Según la Prueba t student

** Diferencia significativa $(p<0.05)$

Tabla 10. Puntajes según el método de Metcoff de los recién nacidos nutridos vs. desnutridos. Servicio de Neonatología del Hospital de Sullana II-2 MINSA. Diciembre 2011 - Enero 2012.

\begin{tabular}{|c||c|c|c|c|c|c|c|c|c|c|c|c||c||c||}
\hline \multicolumn{10}{||c||}{ Evaluación clínica del estado nutricional } \\
\hline \hline \\
$\begin{array}{c}\text { Puntaje } \\
\text { de } \\
\text { Metcoff }\end{array}$
\end{tabular}

Fuente: Base de datos

\section{DISCUSIÓN}

La mayoría de estudios que utilizan y evalúan el estado nutricional de un recién nacido se refieren a un parámetro antropométrico: el peso, o relacionan el peso, talla y perímetro cefálico con la edad gestacional, identificando el momento en que se afecta el crecimiento fetal y la etiología de los diferentes tipos de retardo de crecimiento intrauterino. Sin embargo, existen otros parámetros antropométricos que valoran en el $\mathrm{RN}$ los compartimientos graso y proteico, que permiten detectar eventos que ocurrieron en el último trimestre del embarazo, y que al llegar a término ocasionan una disminución del flujo sanguíneo uterino y por ende menor entrega de nutrientes y de oxígeno al feto. Si bien los parámetros antropométricos (peso, talla y perímetro cefálico) pueden ser normales, algunos $\mathrm{RN}$ a término (RNT) adecuados para su edad gestacional (AEG) sufren de desnutrición fetal cuando se valoran los compartimientos graso y proteico a través del test de Metcoff. La intervención temprana en los casos de desnutrición identificada desde la etapa neonatal puede ayudar a disminuir la desnutrición en menores de 5 años (1-6).

El ambiente materno es el principal determinante del peso al nacer: el bajo índice de masa muscular pregestacional, la pobre ganancia de peso en la gestación, entre otros. En países en desarrollo, se reconoce a la desnutrición materna antes y durante la gestación como el principal factor determinante $(15,18)$

La desnutrición fetal altera la composición corporal, reduce la masa muscular, el contenido proteico de los tejidos, deteriorando enzimas y funciones metabólicas, dejando marcas permanentes en la fisiología celular y molecular y en la morfología de ciertos órganos y tejidos in 
utero que pueden aumentar permanentemente la susceptibilidad a enfermedades crónicas en la vida adulta. Este es un diagnóstico clínico totalmente independiente del peso al nacer del neonato, es una consecuencia de una inadecuada ingesta materna de alimentos o del inadecuado transporte de nutrientes (1-7,15).

A pesar de los progresos en la esfera socioeconómica y en el cuidado obstétrico prenatal en los países desarrollados, la tasa de nacimientos de Peso Bajo al Nacer (PBN) se mantiene en alrededor del 6\% (13). El CLAP ha estimado en promedio una incidencia de $11 \%$ de RNBP en Latinoamérica (14). En el Perú esta cifra alcanza valores del $9 \%$ del total de nacimientos. Esto representa unos 60,000 niños que demandan asistencia preferencial y que presentan un riesgo elevado de muerte o de morbilidad a corto o largo plazo (19).

La incidencia de desnutrición clínica en recién nacidos a término adecuados para la edad gestacional en el servicio de neonatología del hospital de Sullana fue de $16 \%$. Estudio realizado por Zubiate en el Instituto Materno Perinatal de Lima encontró una incidencia del 8.9\% (10), frente al $35.7 \%$ encontrado por Romano en Venezuela (11). En Nigeria Adebami (20), encontró que 83 (18.8\%) de los RN tuvieron DN fetal. En un hospital de México, Ortiz (21). evaluó 460 RNT, con el Test de Metcoff encontró: Prevalencia global (AEG, PEG, GEG) $=28.9 \%$; $30 \%$ de los AEG estaban desnutridos. y $79.4 \%$ de los PEG estaban desnutridos.

En relación a la edad materna más de la mitad de recién nacidos de cada grupo corresponden a madres adultas (20-34 años), 53.3\% para los desnutridos frente al $75.6 \%$ para los nutridos; sin embargo, comparamos las 2 variables en estudio y le aplicamos una prueba estadística significativa, encontramos que el mayor valor porcentual con diferencia significativa es la edad materna <19 años (adolescentes). En contraste Zubiate $(9,10)$ encontró que, el $100 \%$ de los recién nacidos desnutridos correspondían a madres adultas, así mismo no hubo ningún desnutrido en madres menores de 20 años. Frente a estos resultados este autor afirma que la edad adulta no sería un antecedente de riesgo para desnutrición clínica $(10,17)$.

En cuanto a la talla materna, el mayor porcentaje se ubica en los recién nacidos de madres con talla $<1.50 \mathrm{~cm}, 35.9 \%$ para los nutridos frente al $53.3 \%$ de los desnutridos; es decir de los 15 recién nacidos con desnutrición clínica, 8 son hijos de madres con esta talla; no coincidiendo con el estudio de Zubiate $(9,10)$ en donde el mayor porcentaje de nutridos $y$ desnutridos pertenecían a madres con talla entre $(1.51-1.55 \mathrm{~cm})$. Se encontraron 5 recién nacidos $(6.4 \%)$ en madres con talla $>1.60 \mathrm{~cm}$ a diferencia del grupo de los desnutridos en el que no se encontró ninguno. No hay relación significativa entre estas variables $(\mathrm{P}=0.848)$.

Con respecto al IMC pregestacional el mayor porcentaje de recién nacidos de cada grupo, $66.7 \%$ para los nutridos y $69.2 \%$ de los desnutridos corresponden a madres cuyo peso pregestacional fue ideal (IMC: 19.8-26). No hay relación significativa entre el IM pregestacional y el estado nutricional según Metcoff $(\mathrm{P}=0.814)$.

En cuanto a la ganancia ponderal materna; si bien el mayor porcentaje de los desnutridos se ubican fuera del rango normal (GP: 9-12 kg), el $33.3 \%$ en madres con ganancia ponderal < a $9 \mathrm{~kg}$ y el $40 \%$ en madres con ganancia ponderal mayor de $12 \mathrm{~kg}$, al compararlo con el grupo de los nutridos, no se encontró una relación significativa entre las variables $(\mathrm{P}=0.841)$.

No se encontró relación significativa entre estado civil materno y el estado nutricional del recién nacido según Metcoff $(\mathrm{P}=0.999)$, si bien, la mayor cantidad de desnutridos corresponde a recién nacidos de madres convivientes $60 \%$, esta cifra es casi similar al $61.5 \%$ de los nutridos. Esto determina que el estado civil no es un factor influyente en la desnutrición fetal.

En relación al grado de instrucción, más del $50 \%$ de recién nacidos de cada grupo (nutridos y desnutridos según Metcoff) corresponden a madres del nivel secundario, seguido del nivel primario con un $33.3 \%$ para los desnutridos y $19.2 \%$ para los nutridos; sin embargo, no una hay relación significativa entre las variables $(\mathrm{P}=0.841)$.

Al analizar el lugar de procedencia de la madre, se encontró una relación significativa entre esta variable y el estado nutricional del recién nacido según Metcoff ( $\mathrm{P}=0.012)$. El $60 \%$ de los desnutridos corresponden a madres de procedencia rural frente al $24.4 \%$ de los nutridos, de la misma manera el $75.6 \%$ de los nutridos corresponden a madres de procedencia urbana frente $40 \%$ de los desnutridos. Esto significa que en este estudio la procedencia materna es un factor influyente en el estado nutricional del recién nacido.

En cuanto a la paridad materna, no se encontró una relación significativa entre esta variable y el estado nutricional del recién nacido según Metcoff $(\mathrm{P}=0.124)$; para ambos grupos el mayor porcentaje de se ubicó en recién nacidos de madres multíparas, $74.4 \%$ para los nutridos y $53.3 \%$ para los desnutridos. En un estudio realizado Zubiate en el instituto materno perinatal 
de Lima (10), la paridad tampoco fue un factor influyente en estado nutricional del recién nacido.

En relación a los controles prenatales, tampoco se encontró relación significativa con estado nutricional del recién nacido según Metcoff $(\mathrm{P}=0.837)$. El $86.7 \%$ de los desnutridos son recién nacidos de madres controladas, cifra que coincide con el estudio de Zubiate (5) en donde encontraron que el $80 \%$ de los desnutridos correspondían a madres con CPN. En cuanto a los recién nacidos de madres no controladas corresponde el $13.3 \%$ para los desnutridos frente al $15.4 \%$ de los nutridos, no habiendo relación significativa que lo hagan un factor influyente, concordando nuevamente con los resultados de este autor. En el mismo grupo de Nigeria, Adebami estudió los factores maternos asociados a DNF, hallando: Inadecuado control prenatal, edad materna $<18$ años, primiparidad, malnutrición materna (IMC < $18.5 \mathrm{~kg} / \mathrm{m}^{2}$ y PB $<23.5 \mathrm{~cm}$ ), bajo nivel socioeconómico, HTA inducida por el embarazo, hemorragia anteparto, infecciones maternas especialmente malaria, ITU y HIV (p < 0.05) (22).

La alteración de los compartimientos graso y proteico podría deberse a un problema de nutrición materna en la última semana de gestación, sin alteraciones en los parámetros antropométricos, aunque siendo el peso AEG, el promedio de peso en el grupo de desnutridos fue significativamente menor que en el grupo de nutridos $(\mathrm{p}=0.001)$ al igual que el perímetro braquial $(\mathrm{p}=0.001)$. Estos resultados concuerdan con los obtenidos en el estudio de Zubiate (10), donde encontraron una diferencia significativa en el peso del grupo de los desnutridos $(\mathrm{P}=0.047)$, contrariamente Romano en un estudio realizado en Venezuela, no encontró diferencia significativa entre peso y braquial (11).

Respecto al sexo del recién nacido en relación al estado nutricional, no se encontró relación significativa estas variables $(\mathrm{P}=0.260)$, sin embargo, Aldana en Guatemala refiere que el sexo masculino es más frecuente en los desnutridos con diferencias estadísticas significativas (13).

Al realizar la distribución de frecuencias con respecto a la calificación obtenida en la evaluación clínica nutricional, el mayor porcentaje de desnutridos tuvieron un puntaje de 23 ( 7 casos), cifra que coincide con Aldana (13), el límite inferior en este grupo fue 19 puntos (1 caso) a diferencia de este autor que encontró recién nacidos con un puntaje de 17 . El mayor porcentaje de nutridos tienen un puntaje de28. Las calificaciones de 25 y 26, que se encuentran en el límite corresponden al $10 \%$ del total de recién nacidos, los que podrían catalogarse como recién nacidos de riesgo para desnutrición en la vida postnatal.

\section{CONCLUSIONES}

En cuanto a las características maternas, hubo relación significativa con a la edad materna (madres adolescentes) y los desnutridos, así mismo con la zona de procedencia de la madre, 6 de cada 10 recién desnutridos corresponden a madres de procedencia rural; no hubo relación significativa entre la talla, IMC pregestacional, ganancia ponderal, estado civil, grado de instrucción, paridad y controles prenatales de la madre y el estado nutricional del recién nacido. Por otro lado, el promedio y desviación estándar de peso y perímetro braquial de los desnutridos fue significativamente menor que en el grupo de los nutridos $(\mathrm{P}=0.001)$. Asimismo, la incidencia de desnutrición clínica en recién nacidos a término adecuados para la edad gestacional mediante el método de Metcoff en el hospital de Sullana es 16 $\%$. Además, durante los meses diciembre 2009 y enero 2010 hubieron 432 recién nacidos vivos, siendo 54 prematuros (12.5\%) y 378 a término $(87.5 \%)$, de estos últimos $3.7 \%$ son PEG, $94.4 \%$ son AEG y $9 \%$ GEG. Finalmente, la evaluación clínica del estado nutricional según Metcoff, es un método fácil, sencillo que ayuda a identificar los signos clínicos de desnutrición en el neonato.

\section{AGRADECIMIENTOS}

A los miembros del Servicio de Neonatología del departamento de Pediatría del Hospital de Sullana, II-2 MINSA por habernos brindado todas las facilidades.

\section{REFERENCIAS BIBLIOGRÁFICAS}

1. TAEUSCH \& BALLARD. Tratado de Neonatologia de Avery. 7ma edición.

2. TICONA, M., Huanco, D., Oliveros, M., Pacora, P.: Crecimiento Fetal y NeoNatal en el Perú. Implicancias en Salud Pública Imprenta Reynoso E.I.R.L. Tacna. Consejo Nacional de Ciencia, tecnología e Innovación Tecnológica 2007

3. TAPIA, José Luis \& VENTURA-JUNCÁ, Patricio: Manual de Neonatologia. 2A EDICIÓN. Santiago, Publicaciones Técnicas Mediterráneo, 2000.

4. KLIEGMAN, R., et al: Nelson Tratado de pediatría 18 ed. 2008 
5. CRUZ, M.: Tratado de Pediatría. 2007. Océano Madrid

6. BARRERAS Salcedo, José Ignacio et al Manual de Neonatología. "Hospital General de Culiacán" 2008

7. DOIG, Jeannette: Evaluación Nutricional del Recién Nacido. Revista SPP - 2004 - nº 2: 3641

8. RONDÓN, Samuel. Recién nacido de bajo peso

http://www.drrondonpediatra.com/recien_nac ido_bajo_peso.htm [Consulta: 27 febrero 2012].

9. VÁZQUEZ, J., Hernández, Y., Farnot, U.: Caracterización de la madre del recién nacido con crecimiento intrauterino retardado: Rev Cubana Obstet Ginecol 2003;29(2)

10.ZUBIATE M., Doig, J., Marcelo, A., Arias, J. Valoración del estado nutricional de recién nacidos a término y adecuados para su edad gestacional por el método de Metcoff en el Instituto Materno Perinatal de Lima: Rev. Perú. Ped. 2006; 59(2): 14-20

11.ROMANO, F., Barbella, S. Callegari, C., Kolster, C.: Evaluación nutricional del recién nacido a término: aplicación de una metodología clínica para diferenciar desnutrición fetal y pequeño para la edad Gestacional: Arch. Venez. Pueric. Pediatr. 2003; 66(4):8-15.

12. VELÁZZUEZ, D., Porto, S., Santana, S.:. La encuesta de Metcoff como instrumento en la evaluación nutricional del recién nacido prematuro: Rev Cubana Pediatr. 2007; 79(1).

13. ALDANA Dardón, Marie Josée. Incidencia de desnutrición fetal en neonatos: estudio prospectivo - descriptivo en el Área de Neonatología del Hospital General San Juan de Dios: [Trabajo de tesis para optar el título de Nutricionistaᄀ] Universidad Francisco Marroquin-Guatemala, Escuela de Nutrición. 2000: 1 - 64

14.GUTIÉRREZ, S.: Valoración de desnutrición intrauterina por medio del método de CANSCORE en recién nacidos a término con peso adecuado para edad gestacional XXIV congreso de investigación biomédica. 2007. México

15. TICONA, M., Huanco, D.: MORTALIDAD PERINATAL HOSPITALARIA EN EL PERÚ: FACTORES DE RIESGO. Rev. chil. obstet. ginecol. 2005, vol.70, n.5, pp. 313-317

16. CHIONG Acosta, Elizabeth Yolanda \& Eguiluz Loiza, Oscar Augusto. Factores Perinatales Morbimortalidad y Estructuración de las Curvas de Crecimiento Post Natal del Recién Nacido Prematuro de muy Bajo Peso (menor de 1500 gr) en el Hospital Daniel Alcides Carrión del Callo durante los años 2001 y 2002. Trabajo de Investigación (Especialista en Pediatría)--Universidad Nacional Mayor de San Marcos. Facultad de Medicina Humana. Escuela de Post-Grado, 2003

17. DEL ÁGUILA, C.: Repercusiones en el crecimiento y desarrollo del niño: Art Ginecol Obstet. (Perú) 1995; 41 (3): 28-1.

18. FANEIT, Pedro; RIVERA, Clara; GONZALEZ, María et al. Estudio nutricional de la embarazada y su neonato: RevObstetGinecolVenez. [En línea]. 2003; 63 (2): 67-74 Disponible en la World Wide Web: <http://www.scielo.org.ve/scielo.php?script=s ci_arttext\&pid=S0048-

$77322003000200002 \& \operatorname{lng}=e s \& n r m=i s o>$. ISSN 0048-7732. [Consulta 24 de enero 2012].

19.Estadísticas nacionales y mapas [artículo en línea]. Datos de desnutrición crónica 2009. <http://peru.nutrinet.org/estadisticasnacionales/indicadores-de-salud-ynutricion/desnutricion-cronica/datos-2009>

20.ADEBAMI O. et. Al., : Prevalence and problems of foetal malnutrition in term babies ay Wesley Guild Hospital, South Western, Nigeria. West Afr. J. Med 2007 OctDec;26(4):278-82

21.ORTIZ M, et al: Prevalencia de la desnutrición fetal en recién nacidos a término. Pediatría de México. Vol 13. N $^{\circ}$ 12. 2011. Pags. $65-70$

22. ADEBAMI OJ., et al.,: Maternal factors in the etiology of fetal malnutrition in Nigeria. Pediatr Int. 2007 Apr;49(2):150-5. 